\title{
À l'aube de la bibliographie : les références externes dans les dictionnaires latins, 1480-1545
}

MARTINE FURNO

Université Stendhal - Grenoble 3

The appearance of printed text resulted in changes to the way text is accessed, which leads to the question of whether these changes modified scholarly practice; and if so, how? The following article examines this question in a particular context-that of dictionaries and encyclopedias, referencing classical citations as guarantors and examples. The article defines the evolution of three important witnesses in this domain from the end of the fifteenth century to the first part of the sixteenth: the Cornu Copiæ by Niccolo Perotti, the Commentarii linguae latinæ by Etienne Dolet, of which two tomes appeared in 1536 and 1538, and the Thesaurus Latinae Linguae by Robert Estienne, in its three versions from 1531, 1536, and 1543. These three books represent the same type of object: language dictionaries, aimed towards a specific public of advanced learners, or the learned. In fact, the material weight of book fabrication, the difficulty from then on of collecting masses of texts, the new study conditions, the sheer quantity of texts available, and the famous temptation of universality, made the practice of regulation and of precise referencing an urgent necessity. The position of learned printers of Estienne's generation was crucial: they helped create and then make commonplace certain material norms that are now so much part of out intellectual habit that we sometimes forget their material origin.

L a question que je me propose de traiter dans cette réflexion sur l'évolution de la bibliographie relève autant de l'histoire de cette ars que de l'histoire du livre : je me suis en effet demandé si, ou comment, le passage à l'imprimé et les modifications qu'il suscite dans l'accès au texte modifient la pratique érudite, notamment dans le cas particulier, pour les dictionnaires et encyclopédies, du référencement des citations classiques produites comme garants et exemples. J'ai tenté de cerner les évolutions de trois témoins importants en ce domaine 
pour la période de la fin du XVe siècle et de la première partie du XVI ${ }^{e}$ siècle : le Cornu Copiae de Niccolò Perotti, les Commentarii linguae latinae d'Etienne Dolet dont les deux tomes paraissent en 1536 et 1538, et le Thesaurus Latinae Linguae de Robert Estienne, dans ses trois versions de 1531, 1536 et 1543. Je me suis limitée à ces trois livres parce qu'ils représentent un même type d'objets, validement comparables entre eux, c'est-à-dire des dictionnaires de langue, qui, même s'ils ne sont pas bâtis sur les mêmes principes, visent de fait, quels que soient leurs discours publicitaires, un même public d'apprenants avancés, voire de doctes.

J'examinerai donc d'abord les pratiques de référencement des citations, et le discours théorique sur ces pratiques, quand il existe ; puis je tenterai de comprendre à quoi tiennent les différences, réelles mais peut-être différemment marquées qu'on ne l'attendrait, que révèle cette petite chasse doxographique sur la fabrique des outils d'apprentissage.

\section{Les testimonia classiques et leur référence : évolution des pratiques et des discours}

Le Cornu Copiae de Niccolò Perotti est un gros ouvrage foisonnant, rédigé et mis au point par son auteur dans les dernières années de sa vie, c'est-à-dire entre 1477 et 1480, mais qui est sans doute fondé sur toute une activité bien antérieure d'excerpta au cours d'innombrables lectures latines ${ }^{1}$. Publié à titre posthume en 1489 à Venise chez Paganino de' Paganini², il a cependant été préparé par l'auteur et ses secrétaires sous la forme d'un manuscrit d'apparat, destiné à être offert à Federico di Montefeltro ${ }^{3}$. En effet, si Perotti n'ignore pas le novum scribendi genus qu' est l'imprimerie, puisqu'il a lui-même procuré des éditions de Pline et Martial et publié une grammaire, tous ouvrages sortis en 1473 , il n'en semble pas moins méfiant ou prudent vis-à-vis de lui ${ }^{4}$ : de toute évidence, son Cornu Copiae est encore de la génération du manuscrit, car il a été pour l'essentiel fondé sur une documentation de ce type, dont nous avons gardé quelques témoins, entre autres un manuscrit de Vitruve lui ayant appartenu.

Le Cornu Copiae se présente donc sous la forme d'un commentaire du Liber de Spectaculis et du livre I de Martial, commentaire qui prend pour support très littéralement chaque mot du texte ancien, pour en faire le prétexte à une fiche lexicographique comportant l'éclaircissement des sens du mot, mais aussi de ses dérivés et antonymes, le tout illustré de citations reprises à un large 
corpus d'auteurs classiques latins. Pour ces citations, la seule référence donnée est celle du nom de l'auteur ${ }^{6}:$ il est tout à fait exceptionnel que Perotti aille audelà, c'est-à-dire jusqu'au titre de l'œuvre ou à une de ses subdivisions. Il est tout aussi muet sur la source qui lui fournit le texte : nous savons qu'il utilise nombre de relais intermédiaires comme des commentateurs ou compilateurs tardifs, mais les identifier fait partie du travail d'archéologie du texte et n'est jamais possible à partir d'une information directe qui serait donnée par l'auteur lui-même. De même, dans la mesure où Perotti ne présente jamais de discussions proprement philologiques sur les textes qu'il cite, nous n'avons non plus aucune information explicite sur les manuscrits ou les imprimés utilisés. Même lorsqu'il cite des auteurs dont nous connaissons un des manuscrits qu' il a possédés, comme pour Vitruve, il est très difficile d'affirmer hors de tout doute que c'est ce support précis qui est cité dans le Cornu Copiae 7 . Les rares cas qui se distinguent du tout venant de la citation exacte, et au fond banale, sont en général des leçons qui peuvent se trouver dans d'autres témoins, et il est au mieux possible d'affirmer que le texte du Cornu Copiae et le texte du manuscrit appartenant à la bibliothèque de Perotti sont les mêmes; il est impossible de prouver formellement que l'un est copié sur l'autre. Un exemple de cette façon de travailler peut être donné à partir d'une remarque sur le verbe adamare, choisie parmi tant d'autres parce que nous allons en retrouver une discussion dans les Commentarii d'Etienne Dolet :

IV, 26 Composita ab amo fiunt peramo, quod est uehementer amo uel usque ad finem amo, deamo depereo, adamo similiter amatorie amo. 27 Sed quando cum aspiratione scribitur adhamo, idem est quod obligo. Cicero : qui enim serius honores adhamauerunt, uix admittuntur ad eos nec satis commendati multitudini possunt esse.

[26 Les composés de amo sont peramo c'est-à-dire aimer violemment ou aimer jusqu'au bout, deamo dépérir, adamo qui ressemble à aimer d'amour. 27 Mais quand adhamo s'écrit avec une aspiration, le sens est le même qu'obligo (lier, attacher, obliger). Cicéron : ceux en effet qui aiment trop tard les honneurs, y sont admis avec difficulté, et ils ne peuvent pas être assez recommandés à la foule. $]^{8}$ 
Dans ce court passage, nous ne voyons apparaître chez Perotti, ni Valla (Elegantiae V, 37) auquel il reprend la définition adamo similiter amatorie amo, ni Nonius (69), auquel il reprend ensuite la citation de Cicéron (Ac. 2, 11 fragments), ni aucune allusion de type philologique sur la source matérielle à laquelle est reprise le texte. La discussion adamo/adhamo est d'ordre sémantique et définitionnelle ; elle n'est pas philologique et ne met pas en jeu des intermédiaires sur l'autorité desquels il y aurait quelque nécessité de s'appuyer. L'auteur donne son interprétation des textes et des mots, qu'elle lui soit personnelle ou qu'il la fasse personnelle après l'avoir reprise à d'autres, et cette parole se suffit à elle-même. Perotti est encore de la génération de doctes pour lesquels il n'y a pas besoin de découvrir au lecteur les outils de son travail ; il y aurait sans doute même une déperdition de prestige à faire pénétrer le lecteur dans l'officine de fabrication du livre.

La pratique d'Etienne Dolet est différente, mais non radicalement étrangère à celle de Perotti. En effet, les deux ouvrages portent le même nom de Commentarii Linguae latinae, (titre de l'un et sous-titre du Cornu Copiae) et sont bâtis sur une sorte de concaténation des lemmes les uns aux autres, qui refuse tout ordre alphabétique. Mais le corpus de citations est restreint volontairement par Dolet autour de Cicéron et de quelques auteurs canoniques du cicéronianisme militant, comme Plaute, Térence, Salluste, Virgile surtout, et parfois Tite Live ou Quintilien. Sur ce fonds de textes plus étroit, il est possible de donner une référence plus précise que le seul nom d'auteur, comme le fait Perotti. Dans la grande majorité des cas, Dolet fournit l'auteur et le titre de l'œuvre, et pour Cicéron il ajoute parfois une subdivision dans l'œuvre, le chapitre ou le paragraphe. Cette pratique est majoritaire, mais non systématique : on trouve des citations de Cicéron avec le titre seul, d'autres de Térence ou Salluste avec l'auteur seul, d'autres plus curieuses comme une mention de Caesar Libro I sans que l'on puisse savoir de quelle œuvre il s'agit. Ces incohérences tiennent sans doute beaucoup au choix de ses sources par Dolet : les Commentarii, tout monumentaux qu'ils soient, sont souvent une compilation de données antérieures. Quand Dolet reprend Perotti, explicitement ou non, il le fait en général en l'état, c'est-à-dire sans rien ajouter au texte, ce qui explique l'apparition dans les Commentarii de citations avec un seul nom d'auteur : Dolet n'a pas complété la référence livrée par son prédécesseur. Ainsi, pour le lemme adamare, il donne une définition polémique à partir de celle 
de Valla, suivie d'un Appendix également polémique qui discute du texte de Perotti, ou plutôt de la lecture de Cicéron par Perotti :

Lapsus est quoque ridicule in huius uerbi expositione Nic. Perottus, Cornucopiae autor, uir alioqui eruditionis immensae, et de lingua latina ita sua diligentia et labore meritus, ut in eo genere illum Latini omnes unicè complectantur. In hoc tamen uoce grauiter hallucinatus est. Adamare enim amatorie amare, ut Laurentius, interpretatur, idque addit : Adamo idem est quod obligo, quando cum aspiratione scribitur, hoc scilicet modo, Adhamo. Ad hanc significationem probandam, locum hunc Ciceronis affert, et Adhamo cum aspiratione legit. Cicero : qui enim serius honores adhamauerunt, uix admittuntur ad eos nec satis commendati multitudini possunt esse. Animum huc aduerte, Lector, et utri assentiare uide. Perottus enim adhamare hoc Ciceronis loco id significare putat, ut fit, quasi hamis inescatis insidias honoribus facere, aut hamis retinere. Nos uero, Adamare, sine aspiratione legendum censemus, idque pace et uenia doctiorum confirmamus, Adamare, pro concupiscere poni. ${ }^{9}$

[Niccolo Perotti, l'auteur du Cornu Copiae, s'est trompé ridiculement dans l'explication de ce verbe, lui qui est ailleurs un homme d'une immense érudition, et qui a tant mérité de la langue latine par sa diligence et son travail que, dans ce domaine, tous les Latins l'embrassent ensemble. Cependant, sur ce mot, il s'est lourdement illusionné. Il prend adamare en effet, comme Laurent, au sens de aimer d'amour : et il ajoute : adamo a le même sens qu'obligo quand il s'écrit avec une aspiration, soit adhamo. Pour prouver ce sens, il fournit ce passage de Cicéron, et lit adhamo avec une aspiration. Cicéron : ceux en effet qui aiment trop tard les honneurs, y sont admis avec difficulté, et ils ne peuvent pas être assez recommandés à la foule. Prête ici attention, lecteur, et vois avec lequel de nous deux tu es d'accord : en effet Perotti pense que dans ce passage de Cicéron adhamare signifie comme s'il y avait "prendre au piège les honneurs avec des hameçons appâtés ", ou " retenir par des hameçons ». Mais nous, nous pensons qu'il faut lire adamare sans aspiration, et nous le maintenons, avec la permission et la bienveillance de plus savants : adamare est mis pour désirer vivement.] 
Or, l'interprétation donnée par Dolet de la citation des Académiques, pour juste qu'elle soit, ne fait pas moins surgir implicitement un autre débat scientifique. En effet, Dolet a bien vu que l'erreur de Perotti tenait à un choix de lecture, choix qui, transposé en termes techniques, devient le choix philologique d'une leçon du texte aux dépens d'une autre. Mais ce travail philologique amène aussi la question du texte de référence sur lequel s'appuie le lexicographe, et auquel il renvoie. Nos habitudes modernes, dont on verra plus loin où et quand peut-être elles commencent, attendent, dans des cas tels que celui d'adamare, que l'auteur nous indique avec quelle édition il a travaillé. Dans les années 1535-1538 qui sont les années d'élaboration des Commentarii, la formulation d'une telle demande n'est pas incongrue : le corpus classique non seulement est largement imprimé, mais il se met en place aussi pour chaque auteur une sorte de vulgate canonique du découpage et de la numérotation des actes, scènes, livres, chapitres, habitudes en grande partie dues à la diligence des imprimeurs et au plus ou moins grand succès de leurs livres ${ }^{10}$.

Dolet cependant ne donne pas ce renseignement, ou de manière assez vague pour que cela revienne à ne pas le donner - sans toutefois ignorer totalement la question. En effet, à l'extrême fin des liminaires du volume 1 des Commentarii, à la suite de l'index, Dolet insère quelques lignes intitulées " Explication de quelques notes, et avertissement sur quelques autres points ». Il y éclaircit le système des abréviations dans les références aux textes de Cicéron ${ }^{11}$, et l'utilité de l'index alphabétique qu'il introduit bien que cela soit contradictoire avec ses discours sur la rationalité de son exposé continu, et ajoute enfin :

Lectionis uarietatem, quam uel nostratia, uel Aldi, Frobeniiue exemplaria habent, nostro iudicio sequuti sumus, nec uni nos superstitiose addiximus. Quid enim non subinde mutat effrenata quorundam sciolorum audacia? Sed in his tamen ut nostrum liberum fuit iudicium, ita, quae uoles, uel probabis uel repudiabis : nihil enim impedio. ${ }^{12}$

[Pour ce qui est de la différence des leçons, que présentent les exemplaires de chez nous, ou d'Alde ou de Froben, nous les avons suivies selon notre jugement, et nous ne nous sommes pas tenus superstitieusement à une seule. En effet, quels brusques changements ne produit pas l'audace effrénée de certains demi-savants? Mais sur ces questions, de même que 
notre jugement a été libre, de même tu approuveras ou réprouveras ce que tu voudras : je n'empêche rien.]

Je reviendrai plus loin sur les arrière-pensées d'un tel discours ; qu'il nous suffise pour l'instant de constater que les partis-pris bibliographiques, même négatifs, ne peuvent plus, à cette époque, être passés totalement sous silence. Il faut les justifier, fût-ce par une pirouette, qui prouve à elle seule que l'imprimé a fait naitre de nouvelles exigences dans l'univers savant, qu'on accepte ou non d'en tenir compte.

Ces exigences apparaissent plus clairement dans la pratique et le discours de Robert Estienne, au fil des trois éditions de son Thesaurus linguce latinoe en 1531, 1536 et 1543. La lecture de ces trois états du dictionnaire révèle au premier abord une apparente incohérence : certains auteurs sont précisément référencés dès 1531, avec nom, œuvre et subdivision de l'œuvre, alors que d'autres références se limitent au nom de l'auteur. Mais le fait que certaines de ces références minimales se précisent entre 1531 et 1543 nous indique qu'il s'agit là de la manifestation d'un travail qui avance et qui est constamment repris : en fait, Estienne affine son système de références au fur et à mesure que progressent ses propres impressions de classiques, impressions auxquelles il renvoie dans ses dictionnaires.

Les retombées de ces impressions sur l'appareillage des citations sont visibles dans le Thesaurus, et elles sont même soulignées par l'imprimeur lexicographe. Le souhait de donner des références les plus précises possible des textes cités, et la priorité donnée pour ces références aux éditions " maison » est en effet explicite dès la préface du Thesaurus de 1531, où Estienne éclaircit l'appareil donné pour Térence (acte, scène, vers), Plaute (scène et vers) et Pline (numéro de la lettre), en expliquant que "c'est ainsi que nous avons distingué ces trois auteurs par des chiffres quand nous les avons imprimés sur nos presses ", et au naif qui lui demande pourquoi le dictionnaire n'offre pas la même commodité pour tous les auteurs, il répond "Parce qu'ils ne sont pas encore venus sous nos presses $»^{13}$.

Ce parti pris a pour conséquence que les éditions du Thesaurus linguce latince de 1536 puis de 1543 comportent tous les nouveaux auteurs référencés «sur nos presses », qui finissent par représenter l'essentiel du corpus classique latin. À la fin des liminaires du Thesaurus linguce latinoe de 1543, c'est-à-dire au même endroit du livre que Dolet, Estienne s'explique de nouveau sur ces 
références : après avoir repris sous une formulation légèrement différente les remarques données pour Plaute et Térence en 1531, il complète celles sur Pline, et en ajoute de nouvelles pour Cicéron, Varron, Caton, Pline l'Ancien, César, Tite Live, Martial, Perse, Juvénal, Virgile, reliquisque poetis ${ }^{14}$. Presque tous ces textes ont été imprimés ou vont l'être sur les presses d'Estienne autour de $1545^{15}$, et lui-même nous éclaire sur son souci de précision, ou sur les imprécisions qui demeurent pour certains auteurs :

Quos quidem certe versuum numeros, nobis, non tibi Lector, ne erres, notauimus : tantum te admonebunt, eos locos quibus adscribuntur, nos non aliena fide protulisse, sed ut in ipsis authoribus inuenti sunt, posuisse. Si quibus autem non adiiciuntur, scito illa nos ex aliis adscripsisse, nec ipsos in iis authoribus unde citantur, potuisse reperire. In Quintiliani capitibus varia sumus secuti exemplaria : quo fiet ut nos corrupte adscripsisse caput dicere nonnunquam possis. ${ }^{16}$

[Cette numérotation des paragraphes, nous l'avons marquée pour nous, Lecteur, et non pour t'éviter à toi de t'égarer : elle t'avertira seulement que les passages auxquels elle se rapporte n'ont pas été produits sur la foi d'un <intermédiaire> étranger, mais sont cités tels qu'on les a trouvés chez les auteurs eux-mêmes. Les passages où la numérotation n'est pas fournie, sache que nous les avons retranscrits à partir de textes d'autrui, sans pouvoir les retrouver nous-mêmes dans les auteurs d'où ils sont cités. Dans les chapitres de Quintilien, nous avons suivi des exemplaires différents : d'où il se fera que tu pourras quelquefois dire que nous avons donné un numéro de chapitre faux.]

Le procédé est évidemment très habile tant du point de vue commercial, puisque le lecteur du dictionnaire qui veut retrouver les textes est rendu virtuellement prisonnier de la librairie stéphanienne, que du point de vue publicitaire : les citations reprises à ces impressions stéphaniennes sont annoncées comme fiables, faites directement sur la collation des auteurs, tandis que celles reprises ex aliis sont automatiquement signalées par l'absence de références comme potentiellement inexactes. Mais je ne crois pas que ce discours ne soit qu'une stratégie de vente. En effet, dans les Thesaurus linguce latince de 1531 et 1536, le référencement unique des Lettres de Pline 
est justifié par l'argument de la vérification des citations : vt siquis locum ex Dictionario conferre velit, id facere nullo negotio queat, "pour que celui qui veut comparer un passage tiré du Dictionnaire puisse le faire sans aucune peine ", tandis que le système plus complexe mis en place en 1536 pour les Lettres de Cicéron, c'est-à-dire numéro de lettre et paragraphe, est présenté in meum notaui usum, "je l'ai noté pour mon usage ", sans autre précision encore. Ce mélange de publicitaire et de pratique (je pense qu'il faut comprendre in meum usum au sens où ce travail est aussi une aide pour Estienne lui-même en tant que lexicographe et qu'imprimeur au même moment des Lettres de Cicéron) disparaît en fait en 1543, pour arriver au seul commentaire général du référencement pratiqué : nobis, non tibi Lector, ne erres, "pour nous, Lecteur, et non pour t'éviter à toi de t'égarer». Or " pour nous » renvoie bien à ce qui suit, c'est-à-dire à la démonstration de la qualité des éditions d'Estienne. La référence bibliographique n'est plus ici seulement un argument de vente ou une commodité matérielle : elle est en fait la justification de la propriété et de la propreté, si je puis dire, du travail de l'intellectuel. Estienne use désormais de ces références bibliographiques comme nous pourrions le faire, pour garantir la validité de son travail.

La dernière remarque sur Quintilien me semble aller dans le même sens : elle cherche certes à dédouaner l'imprimeur de ce qui pourrait apparaître comme une négligence, mais elle est sans doute aussi l' expression d'un scrupule sincère, ou d'un regret. En effet, dans la même situation que Dolet, c'est-à-dire d'avoir à exercer librement son jugement philologique sur les variantes d'un texte classique, Estienne assume comme lui cette responsabilité savante, mais semble déplorer de ne pouvoir encore fournir une norme et une garantie, là où Dolet se dégageait dans la raillerie de quelques scioli. Les deux textes sont quasi contemporains, et les attitudes intellectuelles bien différentes : je voudrais maintenant tenter d'interpréter les discours ou les silences de Perotti à Estienne, au-delà de leur seule matérialité, dans ce qu'il révèlent de la conception de l'auteur et de la prégnance plus ou moins acceptée que l'imprimé prend dans le travail de chacun. 


\section{De la contrainte et de la liberté de l'auteur : conceptions anciennes et réalités nouvelles}

Les différences que nous avons pu relever entre des textes qui ne sont pas très éloignés les uns des autres, et qui ont le même objet, me semblent bien en effet être dues à la conception même que chacun de nos trois auteurs se fait de son rôle et à la perception qu'il a de son statut, plus qu'à un écart de lectorat visé par les trois ouvrages. Les titres des Cornu Copiae, Commentarii, Thesaurus, nous disent assez tous trois qu'ils visent le foisonnement et l'abondance, et leurs parallèles (même monolinguisme savant, même primauté donnée à l'explication du sens par les testimonia) nous emmènent vers un même public, celui des apprenants avancés, si ce n'est déjà celui des doctes confirmés qui voient dans le dictionnaire un outil commode - et inavoué en général, mais ceci est une autre histoire...

Perotti, au début de notre parcours, travaille en terrain quasi vierge : s'il est loin d'être le premier à se soucier de l'ars scribendi des Anciens, il n'a à sa disposition comme outil d'exercice que des ouvrages comme les Elegantiae de Valla, qui sont davantage ce que nous appellerions une stylistique qu'un lexique. En effet, même si les Elegantiae comportent de nombreuses définitions, elles visent, comme leur titre l'indique, l'écriture élégante, et sont truffées de commentaires grammaticalement normatifs et explicites qu'un lexique ne comporte en général qu'implicitement dans les exemples. Lorsqu'il rassemble son matériel lexicographique, Perotti cherche donc à construire un art latin d'écrire à travers les seuls textes anciens, qui lui fourniront par eux-mêmes, et par leur simple présence, un discours sur la correction et l'élégance n'appelant pas, ou très peu, de métadiscours de sa part. Tout le travail de l'auteur, et tout son poids, se concentrent dans la collection des testimonia, dans le choix de leur forme, et dans le montage de leur présentation : poids peut-être d'autant plus important qu'il n'y a justement pas de parole à la première personne de cet auteur. Derrière ces apparents effacements ou modestie, se cache une conception de l'auteur encore marquée par le Moyen Âge, lui accordant un statut presque omnipotent. Pour Perotti en effet, comme pour les clercs qui l'ont précédé, celui qui a accès aux livres (ici aux textes classiques) se doit d'être un passeur, qui réunit le savoir en un livre autre pour le transmettre aux frères moins favorisés ${ }^{17}$. Mais cela suppose aussi une sorte d'effet de boucle, à savoir que ce lecteur premier des livres qui devient auteur doit lui-même 
être reconnu comme autorité pour être un médiateur de confiance dans cette lecture : autorité qui lui est conférée en fait par le statut même de docte ayant accès aux livres. Nous avons là une conception que l'on pourrait dire verticale de la diffusion du savoir : elle induit ce que nous trouvons dans le Cornu copiae, c'est à dire des testimonia fournis sans autre référence que le nom de l'auteur, lequel est suffisant pour assurer la valeur de la citation compte tenu du but visé. Quant à la forme même de la citation, à sa fiabilité, et à son origine, le lecteur doit s'en remettre à l'autorité du savant compilateur, qui bénéficie a priori d'une science rendue sûre par son existence même. Cette conception est évidemment le propre d'un temps où la rareté de l'accès aux textes confère à cet accès, lorsqu' on le possède, un prestige intrinsèque.

Quelque temps plus tard, on s'attendrait à ce que tout ait changé pour Etienne Dolet. Ce n'est pas tout à fait le cas cependant : quelles que soient les différences contextuelles entre la rédaction du Cornu Copiae et celle des Commentarii, Dolet a d'évidence la même conception verticale du savoir que Perotti, et s'accorde la même position d'architecte omniscient dans la construction de son ouvrage. En effet, les Commentarii entrent dans une polémique, celle du cicéronianisme, dans laquelle Dolet s'est engagé contre Érasme avec le De imitatione Ciceroniana en 1535 : cet ouvrage pamphlétaire comprend des passages entiers des Commentarii, dont ils sont une sorte d'édition princeps ${ }^{18}$. Ce détail nous montre clairement quel est le trajet intellectuel de Dolet, et quel est le statut des Commentarii : venu de l'université, Dolet va vers l'imprimerie, pour laquelle il jouira d'un privilège exceptionnel de la part de François $\mathrm{I}^{\text {er }}$ en 1539, comme on va vers un moyen au service d'une démonstration ou d'une cause. Les Commentarii sont donc un instrument par lequel Dolet veut démontrer la validité d'une conception du cicéronianisme que ses adversaires jugent trop étroite et ridicule ; il s'appuie alors sur un corpus choisi par lui, ordonné par lui, où l'organisation des références relève d'un a priori que cette organisation même est chargée de soutenir. Se limiter dans ce cadre à ce que nous appellerions une « édition de référence» faite par d'autres est impossible : l'utilisation d'un seul texte imprimé et stable devient une contrainte, puisqu'il y a toute chance pour qu'à un moment ou à un autre telle ou telle leçon de cette référence unique ne soit plus cohérente avec l'ensemble de la démonstration. On comprend alors que Dolet refuse de se tenir « superstitieusement » à un texte plutôt qu'à un autre (nec uni nos superstitose addiximus, « et nous ne nous sommes pas tenus superstitieusement à une seule»), ce qui revient évidemment 
à taxer toutes les éditions d'être le produit imparfait de demi-savants, scioli. Il n'est pas étonnant non plus que Dolet récuse simultanément les critiques à venir à travers l'argument de laisser au lecteur la même liberté de jugement que celle qui a été la sienne : quelle que soit la part de rhétorique de ce type de texte, Dolet perçoit bien sans doute qu'il y aurait difficulté à revendiquer pour soi la construction d'une démonstration, privée de compte rendu philologique, sans accepter qu'une contre-critique puisse elle-même être fondée sur un dessein pareillement préalable et omnipotent. La référence bibliographique stricte, régulière et continue est pour Dolet une contrainte intellectuelle qu'il récuse, plus qu'une assurance irréfutable et publique de ses talents.

Le trajet intellectuel et la position de Robert Estienne sont exactement inverses, ce qui explique sans doute l'écart diamétral entre sa pratique et celle de Dolet. Estienne, quelle que soit son érudition immense et réelle, est allé de la mécanique à la doctrina, et l'objet-livre a sans doute pour lui un poids et une matérialité qu'il n'a peut-être pas pour d'autres, puisque moins directement liés à la production d'une presse. C'est le livre même qui s'impose dans le dictionnaire, où il introduit ses numéros de pages, de chapitres, de vers, et dont il devient le centre : les Thesauri expliquent la langue, mais n'ont rien à prouver d'autre en ce domaine que la fiabilité des livres dont ils sont composés. Les titres en quelque sorte parlent d'eux-mêmes : si les Thesauri ne comportent pas de digressions comme les Commentarii, c'est bien parce qu'ils ne sont pas une démonstration, et qu'il n'y a donc pas de déviation possible hors du sujet, mais une collection d'ailleurs centrifuge puisqu'elle réunit le savoir contenu dans les livres en donnant au lecteur les moyens de retourner aux livres. Si l'on ajoute à son statut primordial d'imprimeur le contexte évangélique dans lequel évolue Robert Estienne, on comprend que le livre (et on est tenté alors d'écrire le Livre) n'est pas le moyen d'une démonstration, mais un instrument de liberté intellectuelle et individuelle : les Thesauri, comme je l'ai dit, rassemblent, mais n'enferment pas, ils ouvrent vers chacun des textes et des livres dont ils sont composés, et qui vont pouvoir être lus ou étudiés en rapport les uns avec les autres grâce aux rapprochements que permet le dictionnaire. Pour cela, Estienne doit prouver citation après citation la validité de son travail de lexicographe par la qualité de son travail d'imprimeur : ce sont alors les références bibliographiques systématiquement explicitées et vérifiables qui lui confèrent la même dignité scientifique que celle que leur statut de doctes aux patentes universitaires conféraient à Perotti et Dolet. 


\section{Conclusion}

En guise de conclusion, je ne voudrais pas laisser par ce petit survol une image faussée de l'aube bibliographique. Il va de soi que quel que soit le degré de sympathie, au sens étymologique du terme, qui transparaît ou non dans mes propos pour l'un ou l'autre de ces savants, aucun d'eux n'est historiquement " meilleur » que l'autre. Je ne voudrais pas notamment que la rigueur scientifique que l'on voit s'exercer chez Estienne ne soit surinterprétée dans le sens d'une modernité jumelle de nos habitudes. Son attitude est importante car fondatrice, mais elle n'est pas unique et ne se diffusera que parce que d'autres éprouvent au même moment les mêmes contraintes et nécessités d'une même pratique. Il serait en effet inexact de faire découler cette «scientificité » d'une idée première ou d'une exigence conceptuelle préalable : je crois qu'en fait elle s'impose par le poids matériel de la fabrication du livre, de la difficulté éprouvée à vouloir collectionner désormais des masses de textes disparates dispersés dans des versions manuscrites ou imprimées non normées, non paginées, non numérotées. Ce sont aussi les nouvelles conditions d'étude, la quantité même des textes désormais à disposition, et la fameuse tentation de l'universalité, qui rendent urgentes la pratique d'une régulation et d'un référencement précis sans lesquels l'esprit ne peut plus maîtriser les objets du savoir. C'est pourquoi je crois cruciale la position des imprimeurs savants de la génération d'Estienne. Au carrefour du matériel et de l'intellectuel, ce sont eux qui vont aider à créer puis banaliser certaines normes matérielles qui nous sont devenues tellement évidentes que nous en oublions parfois qu'elles ont été d'abord matérielles, et ne se sont imposées qu'ensuite comme habitudes intellectuelles. Les générations suivantes recommenceront peu à peu à séparer les statuts du docte et du technicien, le premier ayant intégré dans son travail les apports pratiques fournis par le second : mon souci, ici et en d'autres lieux, n’a été que de rendre au plus juste à ces hommes mécaniques la part de ce que les savants de leur temps, autant que nous-même, leur devons. 


\section{Notes}

1. Sur ce livre, voir Martine Furno, Le Cornu Copiae de Niccolò Perotti : culture et méthode d'un humaniste qui aimait les mots (Genève : Droz, coll. Travaux d'humanisme et de Renaissance 294, 1995).

2. Niccolò Perotti, Cornu Copiae (Venise : Paganino de’ Paganini, 1489).

3. Soit l'actuel Urbinas Latinus 301 de la Bibliothèque Apostolique Vaticane (Rome).

4. Perotti rédige, en 1470 probablement, une lettre adressée à Franceso Guarnieri, dans laquelle il manifeste le désir de voir s'établir une sorte de " charte déontologique " pour l'utilisation de ce nouveau mode de diffusion des textes, qui peut à ses yeux se révéler aussi utile que pernicieux s'il tombe en de mauvaises mains. Quelques références sur ce texte dans Furno, Le Cornu Copiae de Niccolò Perotti, p. 126 et quelques autres textes sur la question dans Jean-Louis Charlet, "La bibliothèque et le livre à travers trois témoignages humanistes : N. Perotti, F.M. Grapaldo, Polidoro Virgili », in L'Europa del libro nell'età dell'Umanesimo, éd. Luisa Secchi Tarugi, (Florence : F. Cesati, 2004), p. 79-92. L’expression nouum scribendi genus apparaît dans ce texte pour désigner la presse.

5. Soit l'actuel Palatinus Latinus 1563 de la Bibliothèque Apostolique Vaticane (Rome).

6. Pour plus de détails, voir Furno, Le Cornu Copiae de Niccolò Perotti, p. 52-55.

7. Cf. Martine Furno, «Utilisation du De Architectura de Vitruve dans le Cornu Copiae de Niccolò Perotti », Studi Umanistici Piceni 13 (1993), p. 79-86.

8. Le texte est cité dans l'édition dirigée par Jean-Louis Charlet et établie pour le volume d'où est tiré ce passage par M. Pade et J. Ramminger : N. Perotti, Cornu Copiae seu linguae latinae commentarii (Sassoferrato : Istituto Internazionale di Studi Piceni, 1995) vol. 4. Toutes les traductions des textes latins sont les miennes.

9. Etienne Dolet, Commentarii linguae latinae (Lyon : Gryphe, 1536), vol.1, col. 4.

10. Robert Estienne est particulièrement soucieux de ce genre de commodités nouvelles (voir par exemple son édition de Perse en 1527, son premier classique qui est également la première édition à comporter la numérotation systématique des vers des Satires, une à une), mais on voit apparaître des découpages chez Alde et les Italiens, repris ensuite notamment par des éditeurs comme Bade à Paris, Gryphe à Lyon et Froben à Bâle.

11. Commentarii linguae latinae, $\mathrm{f}^{\circ}\left[\mathrm{Dd} \mathrm{v}^{\circ}\right]$ : Facile enim intelliges Pro Mil. Pro Liga. Pro Flacc. Pro Milone, Pro Ligario, Pro Flacco supponi, [Tu comprendras facilement 
que Pro Mil. Pro Liga. Pro Flacc. sont mis pour Pro Milone, Pro Ligario, Pro Flacco].

12. Il n'existe pas d'édition complète, ni même importante, de Cicéron dans la bibliographie de Johann Froben. On n'en trouve en effet chez cet imprimeur que deux éditions : une des Tusculanae quaestiones per Erasmum diligenter emendatae et scholiis illustratae (Bâle : Johann Froben, novembre 1523 : in- $4^{\circ}$. [8], 221, [1], [2] p. = VD16 ZV 3448) et une de plusieurs dialogues philosophiques, (De officiis, De amicitia, Paradoxa, Somnium Scipionis et De senectute Graeca traductio Theodori Gazae [Bâle : Johann Froben, août 1520] : in $-4^{\circ}$. 40, 347, [1] p. = VD16 C 3175). Je remercie Jean-François Cottier de m'avoir fourni ces précisions. Il n'est pas impossible que Dolet évoque ici de mémoire Cratander ou Herwagen, qui donnent des Opera Omnia de Cicéron respectivement en 1528 et 1534.

13. Cf. Thesaurus Linguae Latinae, 1531, remarques annexes à la préface : "In Terentianis testimoniis ferme ubique tres numeri signati sunt, quorum primus actum significat, secundus scenam, tertius, uersiculum, hoc est, ut uulgus dicit, lineam. In Plautinis duo, prior designat quamlibet comødiae diuisionem, hoc est, uel argumentum, uel prologum, uel scenam, posterior uersiculum indicat. In testimoniis ex Plinii epistolis unicus est numerus, idemque epistolam denotans. Sic enim hos treis authores nostris expressos characteribus per numeros distinximus, ut siquis locum ex Dictionario conferre uelit, id facere nullo negotio queat. At cur, inquiet percontator, non idem fecisti in omnibus authoribus ? Quia nondum sub nostrum praelum uenerunt. » [Les citations de Térence ont presque partout été signalées par trois chiffres : le premier désigne l'acte, le second la scène, le troisième le vers ou, comme on le dit en vernaculaire, la ligne. Dans les citations de Plaute, il y a deux chiffres, le premier désigne n'importe laquelle des divisions de la pièce, c'est-à-dire l'argument, le prologue, ou la scène, et le second indique le vers. Pour les citations tirées des Lettres de Pline, il n'y a qu'un seul chiffre, qui désigne la lettre. En effet, c'est ainsi que nous avons distingués ces trois auteurs par des chiffres quand nous les avons imprimés sur nos presses, de sorte que celui qui veut vérifier un passage tiré du dictionnaire, puisse le faire sans aucune peine. Mais pourquoi, dira le questionneur, n'as-tu pas fait la même chose pour tous les auteurs? Parce qu'ils ne sont pas venus sous nos presses.]

14. Thesaurus Linguae Latinae, 1543, remarques annexes à la préface, Huius operis praecipua sunt commoda : "Terentiana testimonia illud proprium habent, quod ternas numerorum notas ubique illis adiunximus. Quarum prima actum significat : sequens ad scenam pertinet : tertia ad versiculum, quam vulgus lineam 
solet appellare. In Plautinis item testimoniis quamlibet comœdiæ diuisionem, hoc est vel argumentum, vel prologum, vel scenam, numerus prior indicat, posterior ostendit versiculum. In testimoniis ex epistolarum libris C. Plinii Junioris unico numero contenti fuimus qui epistolam indicaret, præpositis literis epist. quibus a suo auunculo Plinio Secundo, qui de Naturali historia scripsit, distingueretur. In M. Tullii epistolis, librum, epistolam, et versum notauimus : in cæeteris eius operibus librum et versum, nonnunquam etiam versum solum, ut in Orationibus, aliisque nonnullis. Idem factum in Catone, Varrone, Columella, Plinio, qui libris et capitibus distincti sunt : Cæsare item, Liuio, et Martiale, Persio, Juuenale, Virgilio, reliquisque poetis. " [Les citations de Térence ont cette particularité propre que nous leur avons ajouté à toutes un appareil de trois chiffres. Le premier signifie l'acte; le second se rapporte à la scène, et le troisième au vers, que l'on appelle en vernaculaire d'habitude la ligne. De même, dans les citations de Plaute, le premier chiffre indique l'une des divisions de la comédie, c'est-à-dire soit l'argument, soit le prologue, soit la scène, et le dernier chiffre montre le vers. Dans les citations tirées des livres de Pline le Jeune, nous nous sommes contentés d'un seul chiffre pour indiquer la lettre, chiffre précédé de la mention epist., qui le distingue de son oncle Pline qui a écrit l'Histoire Naturelle. Pour les Lettres de Cicéron, nous avons marqué le livre, la lettre et le paragraphe ; dans ses autres œuvres, le livre et le paragraphe, quelquefois même le paragraphe seul, comme dans les Discours, et dans quelques autres œuvres. Il a été fait de même dans Caton, Varron, Columelle, Pline, qui sont distingués en livres et chapitres ; de même pour César, Tite Live, et Martial, Perse, Juvénal, Virgile et les autres poètes]. En 1531, le système de référence n'était expliqué que pour Plaute, Térence, et Pline le Jeune ; en 1536, étaient ajoutés à ces trois auteurs les Lettres et les Discours de Cicéron, Caton, Varron, Columelle, Pline l'Ancien, César, Tite Live, et Martial.

15. Des auteurs cités, il n'est que Tite Live, et, si on l'ajoute aux " autres poètes », le Sénèque tragique, dont je ne connaisse pas d'édition par Robert Estienne.

16. Thesaurus Linguae Latinae, 1543, remarques annexes à la préface, Huius operis praecipua sunt commoda.

17. La question du statut et des fonctions de l'auteur compilateur au Moyen Âge a été souvent traitée, voire débattue, mais sans avoir été précisément abordée sous l'angle de la référence bibliographique - et pour cause, la question ne se posant pas en ces termes, me semble-t-il, avant le XVI ${ }^{\mathrm{e}}$ siècle. La conception d'un auteur "vertical " ayant un accès autorisé aux sources est celle par exemple de Vincent de Beauvais dans la présentation du Speculum Maius : voir pour un petit aperçu de 
cette position Furno, Le Cornu Copiae de Niccolò Perotti, p. 84-89. Voir aussi plus généralement Alastair J. Minnis, Medieval Theory of Authorship. Scholastic Literary Attitudes in the Later Middle Ages (Londres : Scolar Press, 1984), et pour une mise en perspective globale avec des temps plus modernes, Ann Moss, Les Recueils de lieux communs : méthode pour apprendre à penser à la Renaissance, traduit de l'anglais par Patricia Eichel-Lojkine, et al. (Genève : Droz, 2002), (traduction de Printed Commonplace-Books and the Structuring of Renaissance Thought [Oxford, New York : Oxford University Press, Clarendon Press, 1996]).

18. Il s'agit pour l'essentiel de digressions sur l'éloquence et sur les arts qui se retrouvent dans les deux textes; pour le détail de ces passages et leur analyse, voir Émile Telle, L'Erasmianus siue Ciceronianus d'Etienne Dolet (Genève : Droz , 1974), p. 54-69. 
